\title{
Dirichlet series expansions of $p$-adic L-functions
}

\author{
Heiko Knospe ${ }^{1}$ Lawrence C. Washington ${ }^{2}$
}

Received: 17 February 2021 / Published online: 30 August 2021

(c) The Author(s) 2021

\begin{abstract}
We study $p$-adic $L$-functions $L_{p}(s, \chi)$ for Dirichlet characters $\chi$. We show that $L_{p}(s, \chi)$ has a Dirichlet series expansion for each regularization parameter $c$ that is prime to $p$ and the conductor of $\chi$. The expansion is proved by transforming a known formula for $p$-adic $L$-functions and by controlling the limiting behavior. A finite number of Euler factors can be factored off in a natural manner from the $p$-adic Dirichlet series. We also provide an alternative proof of the expansion using $p$-adic measures and give an explicit formula for the values of the regularized Bernoulli distribution. The result is particularly simple for $c=2$, where we obtain a Dirichlet series expansion that is similar to the complex case.
\end{abstract}

Keywords p-adic L-Functions · Dirichlet Characters · Dirichlet Series · Euler Factors · Regularized Bernoulli Distributions · p-adic Measures

Mathematics Subject Classification Primary: 11R23 - Secondary: 11R42 - 11S80 - 11M41

\section{Introduction}

Let $p$ be a prime, let $q=p$ if $p$ is odd and $q=4$ if $p=2$, and let $\chi$ be a Dirichlet character of conductor $f$. A $p$-adic L-function $L_{p}(s, \chi)$ for a Dirichlet character $\chi$ is a $p$-adic meromorphic function and an analogue of the complex $L$-function. For powers of the Teichmüller character $\omega$ of conductor $q$, one obtains the $p$-adic zeta functions $\zeta_{p, i}=L_{p}\left(s, \omega^{1-i}\right)$, where $i=0,1, \ldots, p-2(i=0,1$ if $p=2)$. It is well known that $L_{p}(s, \chi)$ is identically zero for odd $\chi$. $p$-adic $L$-functions have a long history and the primary constructions going back to Kubota-Leopoldt [6] and Iwasawa [3] are via the interpolation of special values of complex $L$-functions.

Communicated by Jens Funke.

Heiko Knospe

heiko.knospe@th-koeln.de

Lawrence C. Washington

lcw@umd.edu

1 Faculty 07, TH Köln - University of Applied Sciences, Campus Deutz, Betzdorfer Str. 2, D 50679 Köln, Germany

2 Mathematics Department, University of Maryland, 4176 Campus Dr, College Park, MD 20742-4015, USA 
It can also be shown that $p$-adic $L$-functions are in fact Iwasawa functions.

It is well known that for $\operatorname{Re}(s)>0$,

$$
\left(1-2^{1-s}\right) \zeta(s)=\sum_{n=1}^{\infty} \frac{(-1)^{n+1}}{n^{s}}
$$

and, more generally, if $c \geq 2$ is an integer,

$$
\left(1-\chi(c) c^{1-s}\right) L(s, \chi)=\sum_{n=1}^{\infty} \chi(n) \frac{a_{c, n}}{n^{s}},
$$

where $a_{c, n}=1-c$ if $n \equiv 0 \bmod c$ and $a_{c, n}=1$ if $n \not \equiv 0 \bmod c$. In the following, we derive similar, but slightly different, expansions for $p$-adic $L$-functions.

An explicit formula for $L_{p}(s, \chi)$ is given in [9] (Theorem 5.11): let $F$ be any multiple of $q$ and $f$. Then $L_{p}(s, \chi)$ is a meromorphic function (analytic if $\chi \neq 1$ ) on $\left\{s \in \mathbb{C}_{p}|| s \mid<q p^{-1 /(p-1)}\right\}$ such that

$$
L_{p}(s, \chi)=\frac{1}{F} \frac{1}{s-1} \sum_{\substack{a=1 \\
p \nmid a}}^{F} \chi(a)\langle a\rangle^{1-s} \sum_{j=0}^{\infty}\left(\begin{array}{c}
1-s \\
j
\end{array}\right)\left(\frac{F}{a}\right)^{j} B_{j} .
$$

In Sect. 2, we will use formula (1) to derive a Dirichlet series expansion of $L_{p}(s, \chi)$.

$p$-adic $L$-functions can be also be defined using distributions and measures. Let $\chi$ have conductor $f=d p^{m}$ with $(d, p)=1$. Choose an integer $c \geq 2$, where $(c, d p)=1$. Then there is a measure $E_{1, c}$ on $(\mathbb{Z} / d \mathbb{Z})^{\times} \times \mathbb{Z}_{p}^{\times}$(the regularized Bernoulli distribution) such that

$$
-\left(1-\chi(c)\langle c\rangle^{1-s}\right) L_{p}(s, \chi)=\int_{(\mathbb{Z} / d \mathbb{Z})^{\times} \times \mathbb{Z}_{p}^{\times}} \chi \omega^{-1}(a)\langle a\rangle^{-s} d E_{1, c}
$$

(see [9] Theorem 12.2). In Sect. 3, we give an explicit formula for the values of $E_{1, c}$ and derive the Dirichlet series expansion from (2).

The expansion is particularly simple for $c=2$, and this parameter can be used for $p \neq 2$ and Dirichlet characters with odd conductor. For this case we obtain similar results as in [1, 2], and [4]. In Sect. 4, we provide examples for different parameters $c$.

\section{Expansions of $p$-adic $L$-functions}

First, we derive an approximation of $L_{p}(s, \chi)$ that is close to the original definition of Kubota-Leopoldt (see [6]).

For $r \in \mathbb{C}_{p}^{\times}$we write $\delta(r)$ for a term with $p$-adic absolute value $\leq|r|$.

Proposition 2.1 Let $p$ be a prime number, $\chi$ an even Dirichlet character of conductor $f$, and $F$ a multiple of $q$ and $f$. For $s \in \mathbb{C}_{p}$ with $|s|<q p^{-1 /(p-1)}$, we have 


$$
L_{p}(s, \chi)=\frac{1}{F} \frac{1}{s-1} \sum_{\substack{a=1 \\ p \nmid a}}^{F} \chi(a)\langle a\rangle^{1-s}+\delta(F / q p) .
$$

Proof We use formula (1) above and look at the series $\sum_{j=0}^{\infty}\left(\begin{array}{c}1-s \\ j\end{array}\right)\left(\frac{F}{a}\right)^{j} B_{j}$. The first two terms are $1+(1-s) \frac{-F}{2 a}$. We claim that the $p$-adic absolute value of the other terms $(j \geq 2)$ is less than or equal to $\left|(s-1) F^{2} / q p\right|$. To this end, we note that $|1 / j !| \leq p^{(j-1) /(p-1)}$ and

$$
\left|\left(\begin{array}{c}
1-s \\
j
\end{array}\right)\right| \leq|1-s| p^{(j-1) /(p-1)}\left(q p^{-1 /(p-1)}\right)^{j-1}=|1-s| q^{j-1}
$$

since we assumed that $|s|<q p^{-1 /(p-1)}$. Since $|F| \leq \frac{1}{q},|a|=1$, and $\left|B_{j}\right| \leq p$, we obtain

$$
\left|\left(\begin{array}{c}
1-s \\
j
\end{array}\right)\left(\frac{F}{a}\right)^{j} B_{j}\right| \leq|1-s| q^{j-1} q^{2-j}|F|^{2} p=|1-s||F|^{2} q p .
$$

Then (1) implies

$$
L_{p}(s, \chi)=\frac{1}{F} \frac{1}{s-1} \sum_{\substack{a=1 \\ p \nmid a}}^{F} \chi(a)\langle a\rangle^{1-s}+\frac{1}{2} \sum_{\substack{a=1 \\ p \nmid a}}^{F} \chi \omega^{-1}(a)\langle a\rangle^{-s}+\delta(F / q p) .
$$

It remains to show that the second sum can be absorbed into $\delta(F / q p)$. We have

$$
\begin{aligned}
\sum_{\substack{a=1 \\
p \nmid a}}^{F} \chi \omega^{-1}(a)\langle a\rangle^{-s} & =\sum_{\substack{b=1 \\
p \nmid b}}^{F} \chi \omega^{-1}(F-b)\langle F-b\rangle^{-s} \\
& =-\sum_{\substack{b=1 \\
p \nmid b}}^{F} \chi \omega^{-1}(b)\langle b-F\rangle^{-s} \\
& =-\sum_{\substack{b=1 \\
p \nmid b}}^{F} \chi \omega^{-1}(b)\langle b\rangle^{-s}+\delta\left(F / q p^{-1 /(p-1)}\right) .
\end{aligned}
$$

The last step can be justified by noting that

$$
\frac{\langle b-F\rangle^{-s}}{\langle b\rangle^{-s}}=\left(1-\frac{F}{b}\right)^{-s}=1+\sum_{j=1}^{\infty}\left(\begin{array}{c}
-s \\
j
\end{array}\right)\left(\frac{-F}{b}\right)^{j}=1+\delta\left(F / q p^{-1 /(p-1)}\right),
$$

since $|s|<q p^{-1 /(p-1)}$ (this is the same estimate as earlier, without the presence of the Bernoulli number). This proves the proposition.

Remark 2.2 For $F=f p^{n}$ and $n \rightarrow \infty$, formula (3) gives the original definition of $L_{p}(s, \chi)$ by Kubota and Leopoldt (see [6]).

Remark 2.3 Suppose that $p \neq 2$. Then the error term in the above Proposition (as well as in the following Theorem 2.4) can be improved to $\delta\left(F / p^{2-(p-2) /(p-1)}\right)$. First we note that $B_{j}=0$ for odd $j \geq 3$. By the von Staudt-Clausen Theorem (see [9] 5.10), we have for even $j \geq 2$ : 
$\left|B_{j}\right|=p$ iff $(p-1) \mid j$, and otherwise $\left|B_{j}\right| \leq 1$. Furthermore, $|1 / j !|=p^{\left(j-S_{j}\right) /(p-1)}$, where $S_{j}$ is the sum of the digits of $j$, written to the base $p$ (see [5]). Since $j \equiv S_{j} \bmod (p-1)$, $j \equiv 0 \bmod (p-1)$ is equivalent to $S_{j} \equiv 0 \bmod (p-1)$. We conclude that $\left|B_{j}\right|=p$ yields $S_{j} \geq p-1$ and $|1 / j !| \leq p^{(j-1) /(p-1)} p^{-(p-2) /(p-1)}$. This implies the above error term. We also see that this error term cannot be further improved.

Now we give the Dirichlet expansion of $L_{p}(s, \chi)$. For $m \in \mathbb{N}$, we denote by $\{x\}_{m}$ the unique representative of $x \bmod m \mathbb{Z}$ between 0 and $m-1$.

Theorem 2.4 Let $p$ be a prime number, $\chi$ be an even Dirichlet character of conductor $f$, and $F$ a multiple of $q$ and $f$. Let $c>1$ be an integer satisfying $(c, F)=1$. For $a \in \mathbb{Z}$, define

$$
\epsilon_{a, c, F}=\frac{c-1}{2}-\left\{-a F^{-1}\right\}_{c} \in\left\{-\frac{c-1}{2},-\frac{c-1}{2}+1, \ldots, \frac{c-1}{2}\right\} .
$$

Then we have for $s \in \mathbb{C}_{p}$ with $|s|<q p^{-1 /(p-1)}$ the formula

$$
-\left(1-\chi(c)\langle c\rangle^{1-s}\right) L_{p}(s, \chi)=\sum_{\substack{a=1 \\ p \nmid a}}^{F} \chi \omega^{-1}(a)\langle a\rangle^{-s} \epsilon_{a, c, F}+\delta(F / q p) .
$$

Proof Use (3) with $c F$ in place of $F$, and subtract $\chi(c)\langle c\rangle^{1-s}$ times (3) with $F$, to obtain

$$
\begin{aligned}
\left(1-\chi(c)\langle c\rangle^{1-s}\right) L_{p}(s, \chi) & =\frac{1}{c F} \frac{1}{s-1} \sum_{\substack{a=1 \\
p \nmid a}}^{c F} \chi(a)\langle a\rangle^{1-s} \\
& -\frac{1}{F} \frac{1}{s-1} \sum_{\substack{a=1 \\
p \nmid a}}^{F} \chi(a c)\langle a c\rangle^{1-s}+\delta(F / q p) .
\end{aligned}
$$

Let $0<a_{0}<F$ with $\left(a_{0}, p\right)=1$. Since we assumed $(c, F)=1$ and $p \mid F$, there is a unique number of the form $a_{0} c$ with $0<a_{0} c<c F$ and $\left(a_{0} c, p\right)=1$ in each congruence class modulo $F$ relatively prime to $p$. The first sum in (4) can be written as

$$
\begin{array}{r}
\frac{1}{c F} \frac{1}{s-1} \sum_{\substack{a_{0}=1 \\
p \nmid a_{0}}}^{F} \chi\left(a_{0} c\right)\left\langle a_{0} c\right\rangle^{1-s}\left(\sum_{\substack{a=1 \\
a \equiv a_{0} c \bmod F}}^{c F}\left\langle 1+\frac{a-a_{0} c}{a_{0} c}\right\rangle^{1-s}\right) \\
=\frac{1}{c F} \frac{1}{s-1} \sum_{\substack{a_{0}=1 \\
p \nmid a_{0}}}^{F} \chi\left(a_{0} c\right)\left\langle a_{0} c\right\rangle^{1-s}\left(\sum_{\substack{a=1 \\
a \equiv a_{0} c \bmod F}}^{c F}\left(1+(1-s) \frac{a-a_{0} c}{a_{0} c}\right)\right)+\delta(F / q) .
\end{array}
$$

Note that $\left|\frac{a-a_{0} c}{a_{0} c}\right| \leq|F|$, so this is the same type of estimate used in the proof of Proposition 2.1. Subtracting the second sum in (4) yields 


$$
\begin{aligned}
& \left(1-\chi(c)\langle c\rangle^{1-s}\right) L_{p}(s, \chi) \\
& =\frac{-1}{c F} \sum_{\substack{a_{0}=1 \\
p \nmid a_{0}}}^{F} \chi\left(a_{0} c\right)\left\langle a_{0} c\right\rangle^{1-s}\left(\sum_{\substack{a=1 \\
a \equiv a_{0} c \bmod F}}^{c F} \frac{a-a_{0} c}{a_{0} c}\right)+\delta(F / q p) \\
& =\frac{-1}{c} \sum_{\substack{a_{0}=1 \\
p \nmid a_{0}}}^{F} \chi \omega^{-1}\left(a_{0} c\right)\left\langle a_{0} c\right\rangle^{-s}\left(\sum_{\substack{a=1 \\
a \equiv a_{0} c \bmod F}}^{c F} \frac{a-a_{0} c}{F}\right)+\delta(F / q p) .
\end{aligned}
$$

We compute the inner sum. Let $b=\left\{a_{0} c\right\}_{F}$. Then $a_{0} c=b+\left\{-F^{-1} b\right\}_{c} F$, since the latter sum is congruent to $b$ modulo $F$ and congruent to 0 modulo $c$. If $a$ satisfies $a \equiv a_{0} c \bmod F$ and $0<a<c F$, then $a=b+j F$ with $0 \leq j<c$. Hence

$$
\sum_{\substack{a=1 \\ a_{0} c \bmod F}}^{c F} \frac{a-a_{0} c}{F}=\sum_{j=0}^{c-1}\left(j-\left\{-F^{-1} b\right\}_{c}\right)=c \epsilon_{b, c, F} .
$$

Since $b \equiv a_{0} c \bmod F$, we have $\chi \omega^{-1}(b)\langle b\rangle^{-s}=\chi \omega^{-1}\left(a_{0} c\right)\left\langle a_{0} c\right\rangle^{-s}+\delta(F / q)$ by the same estimate as earlier, so

$$
-\left(1-\chi(c)\langle c\rangle^{1-s}\right) L_{p}(s, \chi)=\sum_{\substack{b=1 \\ p \nmid b}}^{F} \chi \omega^{-1}(b)\langle b\rangle^{-s} \epsilon_{b, c, F}+\delta(F / q p) .
$$

This completes the proof.

We can take the limit of $F=f p^{n}$ as $n \rightarrow \infty$ and obtain:

Corollary 2.5 Let $p$ be a prime number, $\chi$ an even Dirichlet character of conductor $f$, and $c>1$ an integer satisfying $(c, p f)=1$. Then we have for $s \in \mathbb{C}_{p}$ with $|s|<q p^{-1 /(p-1)}$,

$$
-\left(1-\chi(c)\langle c\rangle^{1-s}\right) L_{p}(s, \chi)=\lim _{n \rightarrow \infty} \sum_{\substack{a=1 \\ p \nmid a}}^{f p^{n}} \chi \omega^{-1}(a) \frac{\epsilon_{a, c, f p^{n}}}{\langle a\rangle^{s}} .
$$

The next Theorem shows that a finite number of Euler factors can be factored off in a similar way as in [8], where a weak Euler product was obtained. The main statement is that the remaining Dirichlet series has the expected form, similar to the complex case.

Theorem 2.6 Let $p$ be a prime number and let $\chi$ be an even Dirichlet character of conductor $f$. Let $S$ be any finite (or empty) set of primes not containing $p$ and set $S^{+}=S \cup\{p\}$. Let $F$ be a multiple of $q, f$ and all primes in $S$. Let $c>1$ be an integer satisfying $(c, F)=1$. Then we have for $s \in \mathbb{C}_{p}$ with $|s|<q p^{-1 /(p-1)}$ the formula

$$
-\left(1-\chi(c)\langle c\rangle^{1-s}\right) \cdot \prod_{l \in S}\left(1-\chi \omega^{-1}(l)\langle l\rangle^{-s}\right) \cdot L_{p}(s, \chi)=\sum_{\substack{a=1 \\\left(a, S^{+}\right)=1}}^{F} \chi \omega^{-1}(a) \frac{\epsilon_{a, c, F}}{\langle a\rangle^{s}}+\delta(F / q p) .
$$


Proof We prove the statement by induction on $|S|$. By Theorem 2.4, the formula is true for $S=\varnothing$. Now assume the formula is true for $S$, and $l \neq p$ is a prime with $l \notin S$ and $(c, l)=1$. It suffices to prove the following formula:

$$
\begin{gathered}
\left(1-\chi \omega^{-1}(l)\langle l\rangle^{-s}\right) \sum_{\substack{a=1 \\
\left(a, S^{+}\right)=1}}^{F} \chi \omega^{-1}(a)\langle a\rangle^{-s} \epsilon_{a, c, F}= \\
\sum_{\substack{a=1 \\
\left(a, S^{+} \cup\{l\}\right)=1}}^{l F} \chi \omega^{-1}(a)\langle a\rangle^{-s} \epsilon_{a, c, l F}+\delta(F / q p) .
\end{gathered}
$$

Note that $\left|1-\chi \omega^{-1}(l)\langle l\rangle^{-s}\right| \leq 1$ and $|l F|=|F|$, so we can keep the error term. We can use $l F$ in place of $F$ and write the left side of (5) as

$$
\sum_{\substack{a=1 \\\left(a, S^{+}\right)=1}}^{l F} \chi \omega^{-1}(a)\langle a\rangle^{-s} \epsilon_{a, c, l F}-\sum_{\substack{a=1 \\\left(a, S^{+}\right)=1}}^{F} \chi \omega^{-1}(l a)\langle l a\rangle^{-s} \epsilon_{a, c, F}+\delta(F / q p) .
$$

Now we have

$$
\epsilon_{l a, c, l F}=\frac{c-1}{2}-\left\{-l a(l F)^{-1}\right\}_{c}=\frac{c-1}{2}-\left\{-a F^{-1}\right\}_{c}=\epsilon_{a, c, F} .
$$

Thus (6) is equal to

$$
\begin{aligned}
& \sum_{\substack{a=1 \\
\left(a, S^{+}\right)=1}}^{l F} \chi \omega^{-1}(a)\langle a\rangle^{-s} \epsilon_{a, c, l F}-\sum_{\substack{a=1 \\
\left(a, S^{+}\right)=1}}^{F} \chi \omega^{-1}(l a)\langle l a\rangle^{-s} \epsilon_{l a, c, l F}+\delta(F / q p) \\
& =\sum_{\substack{a=1 \\
\left(a, S^{+}\right)=1 \\
l+a}}^{l F} \chi \omega^{-1}(a)\langle a\rangle^{-s} \epsilon_{a, c, l F}+\delta(F / q p),
\end{aligned}
$$

which shows equation (5).

Remark 2.7 What happens if $S$ contains more and more primes? It is well known that the Euler product does not converge $p$-adically (see [2]), since the factors $\left(1-\chi \omega^{-1}(l)\langle l\rangle^{-s}\right)$ have absolute value $\leq 1$ and do not converge to 1 as $l \rightarrow \infty$. Furthermore, there are infinitely many primes $l$ with $\chi \omega^{-1}(l)=1$ and $\left(1-\langle l\rangle^{-s}\right)^{-1}$ has a pole at $s=0$. We have for $l \neq p$ and $|s|<q p^{-1 /(p-1)}$

$$
1-\langle l\rangle^{-s}=-\sum_{j=1}^{\infty}\left(\begin{array}{c}
-s \\
j
\end{array}\right)(\langle l\rangle-1)^{j} .
$$

The $p$-adic absolute value of each term of the above series is less than

$$
\left(q p^{-1 /(p-1)}\right)^{j} p^{(j-1) /(p-1)} q^{-j}=p^{-1 /(p-1)}<1 .
$$

Hence the product $\prod_{l \in S}\left(1-\chi \omega^{-1}(l)\langle l\rangle^{-s}\right)$ approaches 0 as $S$ expands to include all primes. 


\section{Regularized Bernoulli distributions}

Let $p$ be a prime number and let $d$ be a positive integer with $(d, p)=1$. Define $X_{n}=\left(\mathbb{Z} / d p^{n} \mathbb{Z}\right)$ and $X=\lim _{\longleftarrow}^{\lim } \cong \mathbb{Z} / d \mathbb{Z} \times \mathbb{Z}_{p}$. Let $k \geq 1$ be an integer. Then the Bernoulli distribution $E_{k}$ on $X$ is defined by

$$
E_{k}\left(a+d p^{n} X\right)=\left(d p^{n}\right)^{k-1} \frac{1}{k} B_{k}\left(\frac{\{a\}_{d p^{n}}}{d p^{n}}\right),
$$

where $B_{k}(x)$ is the $k$-th Bernoulli polynomial and $B_{k}=B_{k}(0)$ are the Bernoulli numbers (see $[5,7])$. For $k=1$, one has $B_{1}(x)=x-\frac{1}{2}$. Choose $c \in \mathbb{Z}$ with $c \neq 1$ and $(c, d p)=1$. Then the regularization $E_{k, c}$ of $E_{k}$ is defined by

$$
E_{k, c}\left(a+d p^{n} X\right)=E_{k}\left(a+d p^{n} X\right)-c^{k} E_{k}\left(\left\{\frac{a}{c}\right\}_{d p^{n}}+d p^{n} X\right) .
$$

One shows that the regularized Bernoulli distributions $E_{k, c}$ are measures (see [7]). In the following, we consider only $k=1$; the cases $k \geq 2$ are similar.

Theorem 3.1 Let $p$ be a prime, $c, d \in \mathbb{N}$, and $c \geq 2$ such that $(c, d p)=1$. Let $X$ be as above, and let $E_{1, c}$ be the regularized Bernoulli distribution on $X$. For $a \in\left\{0,1, \ldots, d p^{n}-1\right\}$, we have

$$
E_{1, c}\left(a+d p^{n} X\right)=\frac{c-1}{2}-\left\{-a\left(d p^{n}\right)^{-1}\right\}_{c}=\epsilon_{a, c, d p^{n}} .
$$

Proof By definition,

$$
E_{1, c}\left(a+d p^{n} X\right)=E_{1}\left(a+d p^{n} X\right)-c E_{1}\left(c^{-1} a+d p^{n} X\right)=\frac{a}{d p^{n}}-\frac{1}{2}-c\left(\frac{\left\{c^{-1} a\right\}_{d p^{n}}}{d p^{n}}\right)+\frac{c}{2} .
$$

We give the standard representative of $c^{-1} a \bmod d p^{n}$ :

$$
\left\{c^{-1} a\right\}_{d p^{n}}=\frac{\left\{-a\left(d p^{n}\right)^{-1}\right\}_{c} d p^{n}+a}{c}
$$

Note that the numerator is divisible by $c$, since $\left\{-a\left(d p^{n}\right)^{-1}\right\}_{c} d p^{n} \equiv-a \bmod c$. Hence the quotient is an integer between 0 and $d p^{n}-1$. Furthermore, the numerator is congruent to $a$ modulo $d p^{n}$, and so the quotient has the desired property. We obtain

$$
E_{1, c}\left(a+d p^{n} X\right)=\frac{a}{d p^{n}}+\frac{c-1}{2}-\frac{\left\{-a\left(d p^{n}\right)^{-1}\right\}_{c} d p^{n}+a}{d p^{n}}=\frac{c-1}{2}-\left\{-a\left(d p^{n}\right)^{-1}\right\}_{c}
$$

which is the assertion.

Now the Dirichlet series expansion in Corollary 2.5 follows from Theorem 3.1 and the integral formula (2). 


\section{Expansions for different regularization parameters}

We look at the coefficients $\epsilon_{a, c, d p^{n}}$ for different parameters $c$ and the resulting Dirichlet series expansions. The following observation follows directly from the definition.

Remark 4.1 The sequence of values $E_{1, c}\left(a+d p^{n} X\right)=\epsilon_{a, c, d p^{n}}$ for $a=0,1,2, \ldots, d p^{n}-1$ is periodic with period $c$. The sequence begins with $\frac{c-1}{2}$ and continues with a permutation of $\frac{c-3}{2}, \ldots,-\frac{c-1}{2}$. If we restrict to values of $n$ such that $d p^{n}$ lies in a fixed congruence class modulo $c$, then the values do not change as $n \rightarrow \infty$.

The measure $E_{1, c}$ and the Dirichlet series expansion are particularly simple for $c=2$. Note that we assumed that $d$ and $p$ are odd in this case. If $a$ is even, then $\left\{-a\left(d p^{n}\right)^{-1}\right\}_{2}=0$ and

$$
E_{1,2}\left(a+d p^{n} X\right)=\epsilon_{a, 2, d p^{n}}=\frac{1}{2} .
$$

If $a$ is odd, then $-a\left(d p^{n}\right)^{-1}$ is odd, $\left\{-a\left(d p^{n}\right)^{-1}\right\}_{2}=1$ and

$$
E_{1,2}\left(a+d p^{n} X\right)=\epsilon_{a, 2, d p^{n}}=-\frac{1}{2} .
$$

Hence $E_{1,2}$ is up to the factor $\frac{1}{2}$ equal to the following simple measure:

Definition 4.2 Let $p \neq 2$ be a prime, and let $X \cong \mathbb{Z} / d \mathbb{Z} \times \mathbb{Z}_{p}$ be as above. Then

$$
\mu\left(a+d p^{n} X\right)=(-1)^{\{a\}_{d p^{n}}}
$$

defines a measure on $X$. We call $\mu$ the alternating measure, since the measure of all clopen balls is \pm 1 .

The corresponding integral is also called the fermionic p-adic integral (see [4]).

Now we obtain the following Dirichlet series expansion from Corollary 2.5.

Corollary 4.3 Let $p \neq 2$ be a prime number, and let $\chi$ be an even Dirichlet character of odd conductor $f$. Then we have for $s \in \mathbb{C}_{p}$ with $|s|<p^{(p-2) /(p-1)}$,

$$
\left(1-\chi(2)\langle 2\rangle^{1-s}\right) L_{p}(s, \chi)=\lim _{n \rightarrow \infty} \frac{1}{2} \sum_{\substack{a=1 \\ p \nmid a}}^{f p^{n}}(-1)^{a+1} \chi \omega^{-1}(a) \frac{1}{\langle a\rangle^{s}} .
$$

For $\chi=\omega^{1-i}$ and odd $i=1, \ldots, p-2$, we obtain the branches of the p-adic zeta function:

$$
\zeta_{p, i}(s)=L_{p}\left(s, \omega^{1-i}\right)=\frac{1}{1-\omega(2)^{1-i}\langle 2\rangle^{1-s}} \cdot \lim _{n \rightarrow \infty} \frac{1}{2} \sum_{\substack{a=1 \\ p \nmid a}}^{p^{n}}(-1)^{a+1} \omega(a)^{-i} \frac{1}{\langle a\rangle^{s}}
$$

Remark 4.4 Dirichlet series expansions of $p$-adic $L$-functions were studied by D. Delbourgo in [1] and [2]. He considers Dirichlet characters $\chi$ satisfying $(p, 2 f \phi(f))=1$ and their Teichmüller twists. We obtain the same expansion for $c=2$ and $\chi=\omega^{1-i}$. However, we require $(c, f p)=1$ and use other methods for the proof. 
Similar expansions for a slightly different $p$-adic $L$-function using a fermionic $p$-adic integral (i.e., $c=2$ ) were also obtained by M.-S. Kim and S. Hu (see [4]).

Example 4.5 We look at the case $c=3$. The sequence of values $\epsilon_{a, 3, d p^{n}}$ is periodic with period 3. If $d p^{n} \equiv 1 \bmod 3$, then the sequence is $1,-1,0, \ldots$ If $d p^{n} \equiv 2 \bmod 3$, then we obtain the sequence $1,0,-1, \ldots$.

Corollary 4.6 Let $p$ be a prime number, and let $\chi$ be an even Dirichlet character of conductor $f=d p^{m}$ such that $(3, d p)=1$. If $d \equiv 1 \bmod 3$, then define a sequence $\epsilon_{0}=1, \epsilon_{1}=-1$, $\epsilon_{2}=0, \ldots$ with period 3 . Otherwise, set $\epsilon_{0}=1, \epsilon_{1}=0, \epsilon_{2}=-1$ and extend it with period 3 . Then we have for $s \in \mathbb{C}_{p}$ with $|s|<q p^{-1 /(p-1)}$,

$$
-\left(1-\chi(3)\langle 3\rangle^{1-s}\right) L_{p}(s, \chi)=\lim _{n \rightarrow \infty} \sum_{\substack{a=1 \\ p \nmid a}}^{d p^{2 n}} \chi \omega^{-1}(a) \frac{\epsilon_{a}}{\langle a\rangle^{s}} .
$$

Example 4.7 For $c=5$, we get a periodic sequence with period 5 and we have $\epsilon_{a, 5, d p^{n}}=2$ for $a \equiv 0 \bmod 5$. The next four coefficients are a permutation of the values $-2,-1,0$ and 1 , depending on the class of $d p^{n} \bmod 5$.

Example 4.8 Let $c=7$. Then $\epsilon_{0,7, d p^{n}}=3$. Now suppose, for example, that $d p^{n} \equiv 3 \bmod 7$. Then $\left(d p^{n}\right)^{-1} \equiv 5 \bmod 7$. This yields the values

$$
\epsilon_{1,7, d p^{n}}=1, \epsilon_{2,7, d p^{n}}=-1, \epsilon_{3,7, d p^{n}}=-3, \epsilon_{4,7, d p^{n}}=2, \epsilon_{5,7, d p^{n}}=0, \epsilon_{6,7, d p^{n}}=-2,
$$

and these are extended with period 7.

Acknowledgements The first author thanks Daniel Delbourgo for hints to his work and helpful conversations.

Funding Open Access funding enabled and organized by Projekt DEAL.

Open Access This article is licensed under a Creative Commons Attribution 4.0 International License, which permits use, sharing, adaptation, distribution and reproduction in any medium or format, as long as you give appropriate credit to the original author(s) and the source, provide a link to the Creative Commons licence, and indicate if changes were made. The images or other third party material in this article are included in the article's Creative Commons licence, unless indicated otherwise in a credit line to the material. If material is not included in the article's Creative Commons licence and your intended use is not permitted by statutory regulation or exceeds the permitted use, you will need to obtain permission directly from the copyright holder. To view a copy of this licence, visit http://creativecommons.org/licenses/by/4.0/.

\section{References}

1. Delbourgo, D.: A Dirichlet series expansion for the p-adic zeta-function. J. Aust. Math. Soc. 81, 215224 (2006)

2. Delbourgo, D.: The convergence of Euler products over p-adic number fields. Proc. Edinb. Math. Soc. 52, 583-606 (2009)

3. Iwasawa, K.: On p-adic L-functions. Ann. Math. 89, 198-205 (1969)

4. Kim, M.-S., Su, H.: On p-adic Hurwitz-type Euler zeta functions. J. Number Theory 132, 2977-3015 (2012)

5. Koblitz, N.: p-adic Numbers, p-adic Analysis and Zeta-Functions, volume 58 of Graduate Texts in Mathematics. Springer, Berlin (1984) 
6. Kubota, T., Leopoldt, H. W.: Eine p-adische Theorie der Zetawerte. Teil I: Einführung der p-adischen Dirichletschen L-Funktionen. Journal für die reine und angewandte Mathematik 214, 328-339 (1964)

7. Lang, S.: Cyclotomic Fields I and II, volume 121 of Graduate Texts in Mathematics. Springer, (1990)

8. Washington, L.C.: Euler factors for p-adic L-functions. Mathematika 25(1), 68-75 (1978)

9. Washington, L. C.: Introduction to Cyclotomic Fields, volume 83 of Graduate Texts in Mathematics. Springer, (1997)

Publisher's Note Springer Nature remains neutral with regard to jurisdictional claims in published maps and institutional affiliations. 